\title{
Insecticidal and Repellent Activities of Mimosa pudica L. (Fabaceae) against Cryptolestes pusillus (Schon) (Coleoptera: Cucujidae)
}

\author{
Ujjwal Kumar Mondol and W. Islam* \\ Institute of Biological Sciences, University of Rajshahi, Bangladesh \\ *Corresponding author
}

\section{A B S T R A C T}

\section{Keywords}

Insecticidal, repellent activities, Residual film method, Surface film application method, Mimosa pudica, Cryptolestes pusillus

\section{Article Info}

Accepted: 17 August 2020 Available Online: 10 September 2020

\begin{abstract}
The insecticidal and repellent activities were observed by residual film method and surface film application methods respectively. Leaf, stem and root of Mimosa pudica L. were screened through Petroleum ether, chloroform, ethyl acetate and methanol extracts against flat grain beetle, Cryptolestes pusillus (Schon.). The plant extracts showed less to high mortality by using $0.25,0.50,1.0$ and $2.0 \mathrm{mg} / \mathrm{cm}^{2}$ doses and the root extract showed most potency. In $72 \mathrm{~h}$ of exposure, chloroform extract of root showed the lowest $\mathrm{LD}_{50}$ value $\left(2.024 \mathrm{mg} / \mathrm{cm}^{2}\right)$ and $95 \%$ confidence limit was 1.730-2.292. In case of leaf, stem and root the highest mortality was recorded in chloroform extracts were $73.3,70.0,80.0 \% ; 80.0$, $76.7,90.0 \%$ and $90.0,86.7,96.7 \%$ respectively in 24,48 and $72 \mathrm{~h}$. The repellency response among the three parts of the tested plant was different $(\mathrm{p}<0.05)$ and dose effect was more effective than exposure effect. The root extracts showed strong repellent activity than leaf and stem. The highest F-value was found 189.6542 and 83.48169 for dose and time effect respectively for petroleum ether extract of root. The order of dose-mortality effect of $M$. pudica extracts was root $>$ leaf $>$ stem and the susceptibility of contact toxicity in intensity of solvents was chloroform > petroleum ether $>$ methanol $>$ ethyl acetate. The order of intensity of repellent activity was root (petroleum ether) $>$ leaf (petroleum ether) $>$ stem (ethyl acetate).
\end{abstract}

\section{Introduction}

Mimosa pudica $\mathrm{L}$. is one of the important medicinal plants. It was first formally described by Carl Linnaeus in Species Plantarum in 1753 ('Mimosa pudica'. Australian Plant Name Index (APNI), IBIS database).The generic name Mimosa is derived from the Greek 'mimos' (meaning mimic) alluding to the fact that the leaves move in response to something moving against them. The specific epithet is taken from the Latin word 'pudica' meaning bashful or shrinking to contact (Barneby 1991). Mimosa is a genus of about 400 species of herbs and shrubs, in the sub-family Mimosoideae of the Legume family Fabaceae. M. pudica (Amador-Vegas \& Dominguez 2014) (from Latin: pudica 'shy, bashful or shrinking'; also called sensitive plant, humble plant, sleepy plant, action plant, chuimui, ant-plant ('Mimosa pudica'. Royal Horticultural Society 2018) Dormilones, touch-me-not, shame plant or shy plant) is a 
creeping annual or perennial flowering plant of the pea/legume family Fabaceae.

M. pudica has been used as a traditional medicine for the treatment of neurasthenia, insomnia, traumatic injury, pulmonary tuberculosis and others. It mainly grows on the hillside, jungle, glade and roadside of Asia. Many previous studies on $M$. pudica revealed the presence of flavonoids, phenolics and others (Yuan et al., 2007; Yuan et al., 2007; Yuan et al., 2006; Yuan et al., 2006 and Yuan et al., 2006). In addition, many bioactivities of $M$. pudica were also studied, such as antioxidant, antibacterial, antihepatotoxic activities and so on (Samuel et al., 2008, Nazeema and Brindha 2009, Adhikarimayum et al., 2010, Sadia et al., 2008).

Today, humans are waging an undeclared war against insects in the competitive struggle for existence and almost no crop in the world is free from attack by insects, at least to some degree (Berenbaum, 1995). Farmers have been using plant extracts in pest control for centuries. Botanical insecticides are one of the best alternatives for the hazardous chemical insecticides. Phytochemicals are able to induce different types of abnormalities in insects that could safely be used for insect pest control (Sreelatha and Geetha, 2011).

The protection of stored-grains against insect attack is essential, especially for countries that have inadequate storage facilities and climatic conditions that favor deterioration of grains (Pascual-Villalobos and Robledo 1999). Currently, the control of insect pests is largely dependent on the use of synthetic chemicals in most parts of the world. However, the continuous use of synthetic insecticides is leading to problems such as pest resurgence, resistance and environmental hazards, including human poisoning and toxicity to other non-target organisms (Subramanyam
Hagstrum, 1995; Banwo and Adamu, 2003; Bughio and Wilkins, 2004).

The fascinating results produced by synthetic insecticides against pest, have been overshadowed by recent debates surrounding their hazards to human health and effects on non-target organisms amongst other issues (Adedire et al., 2011). Furthermore, their frequent usages sometimes result in the development of insecticide resistance in target species. The challenge of finding sustainable alternatives to these synthetic insecticides has led to the bio-prospecting of plants with repellent and toxicological properties (Osipitan et al., 2012). Natural products of plant origin have been reported to be useful and desirable tools in pest management because they are effective (Chaubey 2017, Uyi and Obi 2017). Several experiments using plant extracts and powders in human and animal health protection, agriculture and in household pest management have been particularly promising. Although a number of studies have empirically evaluated the use of extracts or powders from several indigenous plant species against insect pests (Habiba et al., 2010, Addisu et al., 2014, Uyi and Osarieme 2016), but little is known about the use of extracts from invasive alien plants to control these noxious species (Uyi and Obi, 2017; Uyi and Osarieme, 2016).

The plant-derived chemicals have been used as potential seed protectant (insecticides and antifeedants) often begins with the screening of plant extracts (Pavela 2007). It is logical to expect biologically active compounds to be produced by plants as a chemical defense measure against their enemies (Oly et al., 2011). The application of plant materials with insecticidal or repellent properties to stored grains is a common traditional method in rural areas around the world (Regnault-Roger et al., 2012, Kedia et al., 2013). 
Cryptolestes pusillus (Coleoptera: Cucujidae) is one of the most harmful insect of stored grains. Stored wheat, wheat flour, rice bran, corn, maize, beans, cassava, cocoa, cowpeas, groundnuts, nere seed, shorghum etc. are attacked by a large number of insect pests and causes a major amount of damage. $C$. pusillus is one of the common and major insect pests of stored grains. It is cosmopolitan in distribution and occurs throughout the whole world especially tropical and subtropical region of the world including Bangladesh. $C$. pusillus completes the life cycle within 30 to 34 days at $30^{\circ} \mathrm{C}$ temperatures. It multiplies rapidly in worm conditions and causes a large amount of damage about $100 \%$ after six months of storage. The damage is caused both by the larval and adult stages of $C$. pusillus.

Natural products from locally available plants with insecticide activity represent a low-cost and sustainable alternative to protect agricultural production. Furthermore, botanical insecticides supposedly pose little threat to the environment or human health compared with synthetic insecticides, and they represent a suitable alternative to controlling mites and insect pests worldwide (Isman 2006, Regnaut-Roger et al., 2012, Kedia et al., 2013).

\section{Materials and Methods}

\section{Plant collection and identification}

The sample plant touch-me-not, Mimosa pudica L. was collected from the Botanical Garden of Rajshahi University Campus. The voucher specimen was then confirmed from the Taxonomical section of the Department of Botany, University of Rajshahi, Bangladesh.

\section{Preparation of extracts}

Sample plants with roots were collected and taken in the laboratory. The soil attached with roots was cleaned by a brush. The leaf, stem and root were separated and the stem and root were chopped in small pieces with the help of scissor and chopper. The leaf, stem and root were taken in wooden trays and kept open in the laboratory for drying in room temperature and tried to keep contamination-free by any cost.

After drying, the samples were taken in a grinding machine and grinded the leaf, stem and root samples separately and preserved in air-tight bags. $100 \mathrm{~g}$ dust of each part (leaf, stem and root) was taken in baspata envelope, sealed and kept them in air-tight transparent polythine bags. The bags were marked with date and preserved for extraction.

\section{Chemical extraction of the plant parts}

To yield the extracts of leaf, $500 \mathrm{~g}$ dust of leaf was taken in five conical flasks of $500 \mathrm{ml}$ (100 g in each conical flask) and poured 400 $\mathrm{ml}$ petroleum ether in each of the five flasks. All the flasks were covered by aluminum foil and shaked for $72 \mathrm{~h}$ in an electric shaker. After shaking the extracts of leaf of petroleum ether was filtered by Whatman filter paper No.1 and collected the extracts in a beaker. After filtering again the flasks were poured by $300 \mathrm{ml}$ petroleum ether, shaked for 48 hours and collected the extract in the same beaker. Repeated the work for third time the beaker was kept at room temperature for evaporation of the solvent. After evaporation, the extract was collected in a glass vial and preserved at $4^{\circ} \mathrm{C}$. The same procedures were followed to yield the extracts of stem and root respectively.

When the extracts of leaf, stem and root of petroleum ether were collected, the dusts in the conical flasks were dried in room temperature to evaporate the solvent. Again the dusts were taken in conical flasks and used chloroform as solvent and followed the same procedures as taken for the petroleum 
ether extracts of leaf, stem and root; taken in glass vials and preserved at $4^{\circ} \mathrm{C}$. The same procedures were followed to yield the ethyl acetate and methanol extracts of leaf, stem and root and preserved at $4{ }^{\circ} \mathrm{C}$ for the future experiments.

\section{Selection of $C$. pusillus}

To carry on the tests for insecticidal activities and also for the repellent potentials of the extracts of M. pudica, Cryptolestes pusillus was selected, because it is an easy cultivable and noble laboratory insect. The life history made this insect as popular choice as test insect for biological studies.

\section{Collection and culture of Cryptolestes pusillus}

Adult beetles of $C$. pusillus were collected from the stock culture of Entomology and Insect Biotechnology Laboratory, Institute of Biological Sciences, University of Rajshahi, Bangladesh and reared as mass-cultures and sub-cultures to be used in the experiments. A standard mixture of sterilized (at $60^{\circ} \mathrm{C}$ for 24 h) cracked wheat and wheat flour with powdered dry yeast in a ratio of 19:1 was used as a food medium in the experiments (Khalequzzaman et al., 1994).

\section{Insecticidal activity test}

The test was carried out by residual film method. $254.34 \mathrm{mg}$ extracts were mixed in 2 $\mathrm{ml}$ of the respective solvents to make stock solutions. From these stock solutions other successive doses were prepared by serial dilution method. Thus four doses were prepared as $0.25,0.5,1.0$ and $2.0 \mathrm{mg} / \mathrm{cm}^{2}$. To conduct the surface film activity test $9.0 \mathrm{~cm}$ Petri dishes were taken for all the doses and their replications. $1 \mathrm{ml}$ of each dose was poured into the lower part of Petri dishes and allowed them to dry out. Then 10 insects were released in each of the Petri dishes. A control experiment by applying the only solvents into the Petri dishes was also set at the same time and three replicates were taken. The whole experiment was observed from time to time and the mortality was counted after every 24 , 48 and $72 \mathrm{~h}$ of exposure. A simple microscope was used to check each beetle by tracing natural movement. In some cases hot needle was taken closer to the bodies (without movement) to confirm death.

\section{Statistical analysis}

The mortality recorded was corrected by the Abbott's (1925) formula in the following manner:

$\mathrm{P}_{\mathrm{r}}=\left\{\left(\mathrm{P}_{\mathrm{o}}-\mathrm{P}_{\mathrm{c}}\right) /\left(100-\mathrm{P}_{\mathrm{c}}\right)\right\} \times 100$

Where, $\mathrm{P}_{\mathrm{r}}=$ Corrected mortality $(\%)$

$\mathrm{P}_{\mathrm{o}}=$ Observed mortality $(\%)$

$\mathrm{P}_{\mathrm{c}}=$ Control mortality $(\%)$, sometimes called natural mortality $(\%)$.

The mortality percentages were subjected to statistical analysis according to Finney (1947) and Busvine (1971) by using software developed in the Department of Agricultural Environmental Science, University of Newcastle upon Tyne, UK. The dosemortality relationship was expressed as median lethal dose $\left(\mathrm{LD}_{50}\right)$.

\section{Repellent activity test}

The repellent activity test was performed by following the surface film application method. A stock solution for each of the petroleum ether, chloroform, ethyl acetate and methanol extracts of leaf, stem and root was prepared. From this stock solution five doses i.e. $0.625,1.25,2.5,5.0$ and $10.0 \mathrm{mg} / \mathrm{ml}$ were made by serial dilution. One control was taken using respective solvent. Half-disc filter papers (Whatman No. 1) were prepared and 
applied doses of all the petroleum ether, chloroform, ethyl acetate and methanol extracts of leaf, stem and root onto half-discs and allowed to dry out in the air. Each treated half-disc then attached lengthwise, edge-toedge to a control half-disc with a scotch-tape and placed in a Petri dish $(9 \mathrm{~cm}$ diam.). Then 10 adult insects were released in the middle of each filter paper circle. Insects were counted on the untreated half of the filter paper disc at one hour interval up to five consecutive hours. Three replications were taken and the averages of the counts were converted to percentage repulsion (PR) using the formula of Talukder and Howse $(1993,1995)$ which was again developed by arcsine transformation for the calculation of ANOVA.

$P R=\left(N_{c}-5\right) \times 20$

Where, $\mathrm{N}_{\mathrm{c}}$ is the number of insects on the untreated half-disc.

Positive values (+) expressed repellency and negative values (-) for attractant activity.

\section{Preparation of doses for repellent activity} of C. pusillus

The repellent activity test used was adopted from the method of McDonald et al., (1970) with some modifications by Talukder and Howse (1993). Half filter paper discs (Whatman No.1, $9.0 \mathrm{~cm}$ diam.) were prepared and $60 \mathrm{mg}$ extract of each sample of different solvents were taken in vials. If the extracts were insoluble in the definite solvent, pure DMSO were added to dissolve the extracts and then $6 \mathrm{ml}$ definite solvent was added with the extracts separately. So the concentration became $60 \mathrm{mg} / 6 \mathrm{ml}$ i.e. $10 \mathrm{mg} / \mathrm{ml}$. From this $6 \mathrm{ml}$ solution, $3 \mathrm{ml}$ were taken to three halfdisc filter papers as $R_{1}, R_{2} \& R_{3}$ each contained $1 \mathrm{ml}$ solution whose concentration was $10 \mathrm{mg} / \mathrm{ml}$. Then $3 \mathrm{ml}$ same solvent were added to the rest $3 \mathrm{ml}$ solution to give the concentration $30 \mathrm{mg} / 6 \mathrm{ml}$, i.e. $5 \mathrm{mg} / \mathrm{ml}$. From this concentration $3 \mathrm{ml}$ were taken to another three half-disc filter papers as $\mathrm{R}_{1}, \mathrm{R}_{2} \& \mathrm{R}_{3}$ each contained $1 \mathrm{ml}$ solution of $5 \mathrm{mg} / \mathrm{ml}$. In the same way $2.5 \mathrm{mg} / \mathrm{ml}, 1.25 \mathrm{mg} / \mathrm{ml}$ and $0.625 \mathrm{mg} / \mathrm{ml}$ doses were prepared and took three replications for each of the doses. The half-disc filter papers with doses were left for sometimes to dry up the solvent in the air. Three half-disc filter papers were soaked with only solvent as control $\left(\mathrm{C}_{1}, \mathrm{C}_{2} \& \mathrm{C}_{3}\right)$ and all the Petri dishes were marked.

\section{Statistical analysis}

Repellency activity test was conducted according to complete randomized experimental design with three replications for each treatment. Data of percentage repulsion (PR) were subjected to arcsine transformation of the proportion and then calculate the ANOVA.

\section{Results and Discussion}

\section{Insecticidal activity}

The test was carried out by residual film method. The effect of different doses of petroleum ether, chloroform, ethyl acetate and methanol extracts of leaf of $M$. pudica on the mortality of $C$. pusillus was shown in Table 1. In case of leaf, the highest activity was found in chloroform extract and the $\mathrm{LD}_{50}$ values were $2.891 \mathrm{mg} / \mathrm{cm}^{2}, 2.472 \mathrm{mg} / \mathrm{cm}^{2}$ and 2.190 $\mathrm{mg} / \mathrm{cm}^{2}$ in 24,48 and $72 \mathrm{~h}$ respectively. The $\mathrm{LD}_{50}$ values of 72 hours of exposure showed significant along with $95 \%$ confidence than 48 and $24 \mathrm{~h}$ of application. The exposure wise dose mortality was $72>48>24 \mathrm{~h}$.

The effect of similar doses of petroleum ether, chloroform, ethyl acetate and methanol extracts of stem of $M$. pudica on the mortality of $C$. pusillus was shown in Table 2. Stem 
extracts were somewhat less toxic than the leaf extracts except the petroleum ether extract. In case of stem, the highest activity was also found in chloroform extract where the $\mathrm{LD}_{50}$ values were $3.144 \mathrm{mg} / \mathrm{cm}^{2}, 2.691$ $\mathrm{mg} / \mathrm{cm}^{2}$ and $2.354 \mathrm{mg} / \mathrm{cm}^{2}$ in 24,48 and 72 hours respectively. Also the $\mathrm{LD}_{50}$ values of 72 hours of exposure showed significant along with $95 \%$ confidence than 48 and 24 hours of application.

Table.1 $\mathrm{LD}_{50}, 95 \%$ confidence limits and regression equations of different extracts of leaf of $M$. pudica against adult $C$. pusillus

\begin{tabular}{|c|c|c|c|c|c|c|}
\hline \multirow{2}{*}{$\begin{array}{l}\text { Test } \\
\text { extracts }\end{array}$} & \multirow{2}{*}{$\begin{array}{l}\text { Time } \\
\text { exposed } \\
\text { (h) }\end{array}$} & \multirow{2}{*}{$\begin{array}{l}\mathrm{LD}_{50} \\
\text { value } \\
\left(\mathrm{mg} / \mathrm{cm}^{2}\right)\end{array}$} & \multicolumn{2}{|c|}{$95 \%$ confidence limits } & \multirow{2}{*}{$\begin{array}{l}\text { Regression } \\
\text { equation }\end{array}$} & \multirow{2}{*}{$\begin{array}{l}X^{2} \text { value } \\
\text { (df) }\end{array}$} \\
\hline & & & $\begin{array}{l}\text { Lower } \\
\text { limit }\end{array}$ & $\begin{array}{l}\text { Upper } \\
\text { limit }\end{array}$ & & \\
\hline \multirow{3}{*}{$\begin{array}{l}\text { Petroleum } \\
\text { ether }\end{array}$} & 24 & 5.560 & 4.296 & 10.250 & $y=-1.37+1.64 x$ & $2.300(3)$ \\
\hline & 48 & 4.535 & 3.674 & 6.711 & $y=-1.13+1.59 x$ & $3.298(3)$ \\
\hline & 72 & 3.870 & 3.206 & 5.134 & $y=-0.92+1.53 x$ & $4.493(3)$ \\
\hline \multirow[t]{3}{*}{ Chloroform } & 24 & 2.891 & 2.442 & 3.409 & $y=-0.75+1.91 x$ & $5.549(3)$ \\
\hline & 48 & 2.472 & 2.074 & 2.866 & $y=-0.48+1.87 x$ & $7.475(3)$ \\
\hline & 72 & 2.190 & 1.854 & 2.503 & $y=-0.55+2.57 x$ & $6.779(3)$ \\
\hline \multirow{3}{*}{$\begin{array}{l}\text { Ethyl } \\
\text { acetate }\end{array}$} & 24 & 4.314 & 3.540 & 6.067 & $y=-1.15+1.72 x$ & $3.166(3)$ \\
\hline & 48 & 3.577 & 2.997 & 4.516 & $y=-0.93+1.69 x$ & $4.356(3)$ \\
\hline & 72 & 2.936 & 2.470 & 3.489 & $y=-0.67+1.71 x$ & $6.026(3)$ \\
\hline \multirow[t]{3}{*}{ Methanol } & 24 & 3.456 & 2.898 & 4.313 & $y=-0.86+1.65 x$ & $4.892(3)$ \\
\hline & 48 & 2.980 & 2.510 & 3.549 & $y=-0.72+1.75 x$ & $5.782(3)$ \\
\hline & 72 & 2.506 & 2.106 & 2.907 & $y=-0.52+1.91 x$ & $7.226(3)$ \\
\hline
\end{tabular}

Table. $2 \mathrm{LD}_{50}, 95 \%$ confidence limits and regression equations of different extracts of stem of M. pudica against adult C. pusillus

\begin{tabular}{|c|c|c|c|c|c|c|}
\hline \multirow{2}{*}{$\begin{array}{l}\text { Test } \\
\text { extracts }\end{array}$} & \multirow{2}{*}{$\begin{array}{l}\text { Time } \\
\text { exposed } \\
\text { (h) }\end{array}$} & \multirow{2}{*}{$\begin{array}{c}\text { LD }_{50} \\
\text { value } \\
\left(\mathrm{mg} / \mathrm{cm}^{2}\right)\end{array}$} & \multicolumn{2}{|c|}{ 95\% confidence limits } & \multirow{2}{*}{$\begin{array}{c}\text { Regression } \\
\text { equation }\end{array}$} & \multirow{2}{*}{$\begin{array}{c}X^{2} \text { value } \\
\text { (df) }\end{array}$} \\
\hline & & & $\begin{array}{c}\text { Lower } \\
\text { limit }\end{array}$ & $\begin{array}{l}\text { Upper } \\
\text { limit }\end{array}$ & & \\
\hline \multirow{3}{*}{$\begin{array}{l}\text { Petroleum } \\
\text { ether }\end{array}$} & 24 & 3.809 & 3.196 & 4.891 & $y=-1.13+1.93 x$ & $3.299(3)$ \\
\hline & 48 & 3.025 & 2.560 & 3.591 & $y=-0.85+1.96 x$ & $4.822(3)$ \\
\hline & 72 & 2.648 & 2.226 & 3.091 & $y=-0.58+1.84 x$ & $6.790(3)$ \\
\hline \multirow[t]{3}{*}{ Chloroform } & 24 & 3.144 & 2.651 & 3.691 & $y=-1.02+2.22 x$ & $3.826(3)$ \\
\hline & 48 & 2.691 & 2.274 & 3.134 & $y=-0.71+2.04 x$ & $5.745(3)$ \\
\hline & 72 & 2.354 & 1.987 & 2.706 & $y=-0.59+2.3 x$ & $7.021(3)$ \\
\hline \multirow{3}{*}{$\begin{array}{l}\text { Ethyl } \\
\text { acetate }\end{array}$} & 24 & 5.085 & 4.013 & 8.459 & $y=-1.23+1.57 x$ & $2.873(3)$ \\
\hline & 48 & 4.435 & 3.573 & 6.614 & $y=-0.94+1.3 x$ & $4.322(3)$ \\
\hline & 72 & 3.576 & 2.946 & 4.668 & $y=-0.58+1.08 x$ & $6.790(3)$ \\
\hline \multirow[t]{3}{*}{ Methanol } & 24 & 4.210 & 3.477 & 5.784 & $y=-1.17+1.79 x$ & $3.014(3)$ \\
\hline & 48 & 3.554 & 2.996 & 4.430 & $y=-1.04+1.91 x$ & $3.826(3)$ \\
\hline & 72 & 2.980 & 2.510 & 3.549 & $y=-0.72+1.75 x$ & $5.782(3)$ \\
\hline
\end{tabular}


Table.3 $\mathrm{LD}_{50}, 95 \%$ confidence limits and regression equations of different extracts of root of $M$. pudica against adult $C$. pusillus

\begin{tabular}{|c|c|c|c|c|c|c|}
\hline \multirow{2}{*}{$\begin{array}{l}\text { Test } \\
\text { extracts }\end{array}$} & \multirow{2}{*}{$\begin{array}{c}\text { Time } \\
\text { exposed } \\
\text { (h) }\end{array}$} & \multirow{2}{*}{$\begin{array}{c}\mathbf{L D}_{50} \text { value } \\
\left(\mathrm{mg} / \mathrm{cm}^{2}\right)\end{array}$} & \multicolumn{2}{|c|}{$95 \%$ confidence limits } & \multirow{2}{*}{$\begin{array}{c}\text { Regression } \\
\text { equation }\end{array}$} & \multirow{2}{*}{$\begin{array}{c}X^{2} \text { value } \\
\text { (df) }\end{array}$} \\
\hline & & & $\begin{array}{c}\text { Lower } \\
\text { limit }\end{array}$ & $\begin{array}{l}\text { Upper } \\
\text { limit }\end{array}$ & & \\
\hline \multirow{3}{*}{$\begin{array}{l}\text { Petroleum } \\
\text { ether }\end{array}$} & 24 & 3.119 & 2.638 & 3.732 & $y=-0.87+1.92 x$ & $4.873(3)$ \\
\hline & 48 & 2.722 & 2.289 & 3.193 & $y=-0.6+1.79 x$ & $7.009(3)$ \\
\hline & 72 & 2.241 & 1.895 & 2.566 & $y=-0.57+2.5 x$ & $7.256(3)$ \\
\hline \multirow[t]{3}{*}{ Chloroform } & 24 & 2.548 & 2.151 & 2.948 & $y=-0.65+2.12 x$ & $6.142(3)$ \\
\hline & 48 & 2.218 & 1.879 & 2.534 & $y=-0.6+2.61 x$ & $6.782(3)$ \\
\hline & 72 & 2.024 & 1.730 & 2.292 & $y=-0.74+3.49 x$ & $6.574(3)$ \\
\hline \multirow[t]{3}{*}{ Ethyl acetate } & 24 & 3.805 & 3.154 & 5.011 & $y=-0.88+1.48 x$ & $4.632(3)$ \\
\hline & 48 & 3.081 & 2.579 & 3.729 & $y=-0.62+1.48 x$ & $6.470(3)$ \\
\hline & 72 & 2.611 & 2.192 & 3.046 & $y=-0.54+1.8 x$ & $7.039(3)$ \\
\hline \multirow[t]{3}{*}{ Methanol } & 24 & 4.649 & 3.705 & 7.274 & $y=-0.96+1.27 x$ & $4.508(3)$ \\
\hline & 48 & 3.765 & 3.103 & 5.000 & $y=-0.7+1.26 x$ & $5.638(3)$ \\
\hline & 72 & 3.184 & 2.662 & 3.897 & $y=-0.64+1.44 x$ & $6.569(3)$ \\
\hline
\end{tabular}

Table.4 $\mathrm{LD}_{50}$ values of different solvent extracts of leaf, stem and root of $M$. pudica in $24 \mathrm{~h}, 48 \mathrm{~h}$ and $72 \mathrm{~h}$ of exposure

\begin{tabular}{|c|c|c|c|c|c|c|c|c|c|}
\hline \multirow{2}{*}{$\begin{array}{l}\text { Test } \\
\text { extracts }\end{array}$} & \multicolumn{3}{|c|}{$24 \mathrm{~h}$} & \multicolumn{3}{|c|}{$48 \mathrm{~h}$} & \multicolumn{3}{|c|}{$72 \mathrm{~h}$} \\
\hline & $\begin{array}{c}\text { Leaf } \\
\text { LD }_{50} \\
\text { value } \\
\left(\mathrm{mg} / \mathrm{cm}^{2}\right)\end{array}$ & $\begin{array}{c}\text { Stem } \\
\text { LD }_{50} \\
\text { value } \\
\left(\mathrm{mg} / \mathrm{cm}^{2}\right)\end{array}$ & $\begin{array}{c}\text { Root } \\
\text { LD }_{50} \\
\text { value } \\
\left(\mathrm{mg} / \mathrm{cm}^{2}\right)\end{array}$ & $\begin{array}{c}\text { Leaf } \\
\mathrm{LD}_{50} \\
\text { value } \\
\left(\mathrm{mg} / \mathrm{cm}^{2}\right)\end{array}$ & $\begin{array}{c}\text { Stem } \\
\mathrm{LD}_{50} \\
\text { value } \\
\left(\mathrm{mg} / \mathrm{cm}^{2}\right)\end{array}$ & $\begin{array}{c}\text { Root } \\
\text { LD }_{50} \\
\text { value } \\
\left(\mathrm{mg} / \mathrm{cm}^{2}\right)\end{array}$ & $\begin{array}{c}\text { Leaf } \\
\text { LD }_{50} \\
\text { value } \\
\left(\mathrm{mg} / \mathrm{cm}^{2}\right)\end{array}$ & $\begin{array}{c}\text { Stem } \\
\mathrm{LD}_{50} \\
\text { value } \\
\left(\mathrm{mg} / \mathrm{cm}^{2}\right)\end{array}$ & $\begin{array}{c}\text { Root } \\
\text { LD }_{50} \\
\text { value } \\
\left(\mathrm{mg} / \mathrm{cm}^{2}\right)\end{array}$ \\
\hline $\begin{array}{l}\text { Petroleum } \\
\text { ether }\end{array}$ & 5.560 & 3.809 & 3.119 & 4.535 & 3.025 & 2.722 & 3.870 & 2.648 & 2.241 \\
\hline Chloroform & 2.891 & 3.144 & 2.548 & 2.472 & 2.691 & 2.218 & 2.190 & 2.354 & 2.024 \\
\hline $\begin{array}{l}\text { Ethyl } \\
\text { acetate }\end{array}$ & 4.314 & 5.085 & 3.805 & 3.577 & 4.435 & 3.081 & 2.936 & 3.576 & 2.611 \\
\hline Methanol & 3.456 & 4.210 & 4.649 & 2.980 & 3.554 & 3.765 & 2.506 & 2.980 & 3.184 \\
\hline
\end{tabular}


Table.5 \% Mortality of Cryptolestes pusillus in different extracts

\begin{tabular}{|c|c|c|c|c|c|c|c|c|c|c|c|c|c|}
\hline \multirow{3}{*}{$\begin{array}{l}\text { Expo } \\
\text { sure } \\
\text { (h) }\end{array}$} & \multirow{3}{*}{$\begin{array}{l}\text { Dose } \\
(\mathbf{m g} / \\
\left.\mathrm{cm}^{2}\right)\end{array}$} & \multicolumn{12}{|c|}{$\%$ Kill } \\
\hline & & \multicolumn{3}{|c|}{ Petroleum ether } & \multicolumn{3}{|c|}{ Chloroform } & \multicolumn{3}{|c|}{ Ethyl acetate } & \multicolumn{3}{|c|}{ Mehtanol } \\
\hline & & Leaf & Stem & Root & Leaf & Stem & Root & Leaf & Stem & Root & Leaf & Stem & Root \\
\hline \multirow[t]{4}{*}{24} & 0.25 & 20.0 & 30.0 & 40.0 & 43.3 & 36.7 & 50.0 & 26.7 & 23.3 & 33.3 & 36.7 & 26.7 & 30.0 \\
\hline & 0.50 & 26.7 & 40.0 & 50.0 & 56.7 & 50.0 & 63.3 & 36.7 & 30.0 & 43.3 & 46.7 & 36.7 & 33.3 \\
\hline & 1.00 & 33.3 & 50.0 & 60.0 & 63.3 & 63.3 & 73.3 & 43.3 & 36.7 & 50.0 & 53.3 & 5.7 & 40.0 \\
\hline & 2.00 & 43.3 & 60.0 & 70.0 & 73 & 70.0 & 80.0 & 53.3 & 46.7 & 56.7 & 63.3 & 3.3 & 50.0 \\
\hline \multirow[t]{4}{*}{48} & 0.25 & 26.7 & 40.0 & 50.0 & 53.3 & 46.7 & 60.0 & 33.3 & 30.0 & 43.3 & 43.3 & 33.3 & 36.7 \\
\hline & 0.50 & 33.3 & 53.3 & 56.7 & 66.7 & 60.0 & 70.0 & 46.7 & 36.7 & 53.3 & 53.3 & 43.3 & 43.3 \\
\hline & 1.00 & 43.3 & 63.3 & 66.7 & 73.3 & 70.0 & 83.3 & 53.3 & 43.3 & 60.0 & 63.3 & 53.3 & 50.0 \\
\hline & 2.00 & 50.0 & 70.0 & 76.7 & 80.0 & 76.7 & 90.0 & 60.0 & 50.0 & 66.7 & 70.0 & 63.3 & 56.7 \\
\hline \multirow[t]{4}{*}{72} & 0.25 & 33.3 & 50.0 & 60.0 & 60.0 & 56.7 & 66.7 & 43.3 & 40.0 & 50.0 & 53.3 & 43.3 & 43.3 \\
\hline & 0.50 & 40.0 & 60.0 & 70.0 & 73.3 & 66.7 & 76.7 & 56.7 & 46.7 & 63.3 & 63.3 & 53.3 & 50.0 \\
\hline & 1.00 & 50.0 & 70.0 & 80.0 & 83.3 & 76.7 & 90.0 & 63.3 & 53.3 & 70.0 & 73.3 & 63.3 & 56.7 \\
\hline & 2.00 & 56.7 & 76.7 & 90.0 & 90.0 & 86.7 & 96.7 & 70.0 & 56.7 & 76.7 & 80.0 & 70.0 & 66.7 \\
\hline
\end{tabular}

Table.6 ANOVA results for repellent activity of leaf extracts of $M$. pudica against adult $C$. pusillus

\begin{tabular}{|l|l|l|l|l|l|l|l|l|}
\hline $\begin{array}{l}\text { Test } \\
\text { material }\end{array}$ & Extracts & $\begin{array}{l}\text { Source of } \\
\text { Variation }\end{array}$ & $\boldsymbol{S S}$ & $\boldsymbol{d f}$ & $\boldsymbol{M S}$ & $\boldsymbol{F}$ & $\boldsymbol{P}$-value & $\boldsymbol{F}$ crit \\
\hline Leaf & $\begin{array}{l}\text { Petroleum } \\
\text { ether }\end{array}$ & Dose effect & 1685.274 & 4 & 421.3184 & 49.74491 & $7.91 \mathrm{E}-09$ & 3.006917 \\
\hline & & Time effect & 578.5058 & 4 & 144.6265 & 17.07599 & $1.26 \mathrm{E}-05$ & 3.006917 \\
\hline & Error & 135.5133 & 16 & 8.469579 & & & \\
\hline & Total & 2399.293 & 24 & & & & \\
\hline & Chloroform & Dose effect & 1821.602 & 4 & 455.4006 & 13.11165 & $6.36 \mathrm{E}-05$ & 3.006917 \\
\hline & Time effect & 332.332 & 4 & 83.083 & 2.392082 & 0.093914 & 3.006917 \\
\hline & Error & 555.7202 & 16 & 34.73251 & & & \\
\hline & Total & 2709.655 & 24 & & & & \\
\hline \multirow{2}{*}{$\begin{array}{l}\text { Ethyl } \\
\text { acetate }\end{array}$} & Dose effect & 1651.455 & 4 & 412.8639 & 19.99361 & $4.57 \mathrm{E}-06$ & 3.006917 \\
\hline & Time effect & 643.0277 & 4 & 160.7569 & 7.784918 & 0.001107 & 3.006917 \\
\hline & Error & 330.3966 & 16 & 20.64979 & & & \\
\hline & Total & 2624.88 & 24 & & & & \\
\hline \multirow{2}{*}{ Methanol } & Dose effect & 1280.406 & 4 & 320.1015 & 46.44371 & $1.31 \mathrm{E}-08$ & 3.006917 \\
\hline & Time effect & 451.473 & 4 & 112.8683 & 16.37612 & $1.64 \mathrm{E}-05$ & 3.006917 \\
\hline & Error & 110.2759 & 16 & 6.892247 & & & \\
\hline & Total & 1842.155 & 24 & & & & \\
\hline
\end{tabular}


Table.7 ANOVA results for repellent activity of stem extracts of $M$. pudica against adult $C$. pusillus

\begin{tabular}{|c|c|c|c|c|c|c|c|c|}
\hline \begin{tabular}{|l} 
Test \\
material
\end{tabular} & Extracts & $\begin{array}{l}\text { Source of } \\
\text { Variation }\end{array}$ & $S S$ & $d f$ & $M S$ & $F$ & P-value & F crit \\
\hline \multirow{16}{*}{ Stem } & \multirow{4}{*}{$\begin{array}{l}\text { Petroleum } \\
\text { ether }\end{array}$} & Dose effect & 1472.515 & 4 & 368.1288 & 13.4846 & $5.38 \mathrm{E}-05$ & 3.006917 \\
\hline & & Time effect & 1052.202 & 4 & 263.0505 & 9.635567 & 0.000365 & 3.006917 \\
\hline & & Error & 436.7991 & 16 & 27.29995 & & & \\
\hline & & Total & 2961.516 & 24 & & & & \\
\hline & \multirow[t]{4}{*}{ Chloroform } & Dose effect & 1800.978 & 4 & 450.2444 & 9.968747 & 0.000303 & 3.006917 \\
\hline & & Time effect & 685.2142 & 4 & 171.3035 & 3.792788 & 0.023581 & 3.006917 \\
\hline & & Error & 722.6496 & 16 & 45.1656 & & & \\
\hline & & Total & 3208.841 & 24 & & & & \\
\hline & \multirow{4}{*}{$\begin{array}{l}\text { Ethyl } \\
\text { acetate }\end{array}$} & Dose effect & 1855.095 & 4 & 463.7739 & 36.56298 & 7.34E-08 & 3.006917 \\
\hline & & Time effect & 925.2233 & 4 & 231.3058 & 18.23568 & $8.29 \mathrm{E}-06$ & 3.006917 \\
\hline & & Error & 202.9479 & 16 & 12.68425 & & & \\
\hline & & Total & 2983.267 & 24 & & & & \\
\hline & \multirow[t]{4}{*}{ Methanol } & Dose effect & 1675.989 & 4 & 418.9973 & 20.1373 & $4.37 \mathrm{E}-06$ & 3.006917 \\
\hline & & Time effect & 1093.728 & 4 & 273.4319 & 13.14133 & $6.27 \mathrm{E}-05$ & 3.006917 \\
\hline & & Error & 332.9124 & 16 & 20.80702 & & & \\
\hline & & Total & 3102.629 & 24 & & & & \\
\hline
\end{tabular}

Table.8 ANOVA results for repellent activity of root extracts of M. pudica against adult $C$. pusillus

\begin{tabular}{|c|c|c|c|c|c|c|c|c|}
\hline $\begin{array}{l}\text { Test } \\
\text { material }\end{array}$ & Extracts & $\begin{array}{l}\text { Source of } \\
\text { Variation }\end{array}$ & $S S$ & $d f$ & $M S$ & $F$ & P-value & F crit \\
\hline \multirow[t]{16}{*}{ Root } & \multirow{4}{*}{$\begin{array}{l}\text { Petroleum } \\
\text { ether }\end{array}$} & Dose effect & 1547.642 & 4 & 386.9106 & 189.6542 & $2.93 \mathrm{E}-13$ & 3.006917 \\
\hline & & Time effect & 681.239 & 4 & 170.3098 & 83.48169 & $1.65 \mathrm{E}-10$ & 3.006917 \\
\hline & & Error & 32.64136 & 16 & 2.040085 & & & \\
\hline & & Total & 2261.523 & 24 & & & & \\
\hline & \multirow[t]{4}{*}{ Chloroform } & Dose effect & 1126.007 & 4 & 281.5017 & 66.00051 & $9.71 \mathrm{E}-10$ & 3.006917 \\
\hline & & Time effect & 530.9797 & 4 & 132.7449 & 31.1232 & $2.29 \mathrm{E}-07$ & 3.006917 \\
\hline & & Error & 68.2423 & 16 & 4.265144 & & & \\
\hline & & Total & 1725.229 & 24 & & & & \\
\hline & \multirow{4}{*}{$\begin{array}{l}\text { Ethyl } \\
\text { acetate }\end{array}$} & Dose effect & 1278.837 & 4 & 319.7093 & 102.7792 & $3.37 \mathrm{E}-11$ & 3.006917 \\
\hline & & Time effect & 581.417 & 4 & 145.3543 & 46.72803 & $1.25 \mathrm{E}-08$ & 3.006917 \\
\hline & & Error & 49.7703 & 16 & 3.110644 & & & \\
\hline & & Total & 1910.025 & 24 & & & & \\
\hline & \multirow[t]{4}{*}{ Methanol } & Dose effect & 1000.317 & 4 & 250.0793 & 127.6997 & $6.34 \mathrm{E}-12$ & 3.006917 \\
\hline & & Time effect & 526.9535 & 4 & 131.7384 & 67.27046 & $8.42 \mathrm{E}-10$ & 3.006917 \\
\hline & & Error & 31.33342 & 16 & 1.958339 & & & \\
\hline & & Total & 1558.604 & 24 & & & & \\
\hline
\end{tabular}


The effect of contact poisoning of root extracts of $M$. pudica against $C$. pusillus showed the highest activity to all the three exposures of application except methanol extract which was shown in Table 3. Among the root extracts, the highest activity was also found in chloroform extract where the $\mathrm{LD}_{50}$ values were $2.548 \mathrm{mg} / \mathrm{cm}^{2}, 2.218 \mathrm{mg} / \mathrm{cm}^{2}$ and $2.024 \mathrm{mg} / \mathrm{cm}^{2}$ in 24, 48 and 72 hours respectively. The recorded $\mathrm{LD}_{50}$ values of 72 hours of exposure observed higher mortality along with $95 \%$ confidence than 48 and 24 hours of application.

Among the extracts of leaf, stem and root, the chloroform extracts of root showed the highest activity. In 24 hours the lowest $\mathrm{LD}_{50}$ value was $2.548 \mathrm{mg} / \mathrm{cm}^{2}$ for chloroform extract of root and the highest was 5.560 $\mathrm{mg} / \mathrm{cm}^{2}$ for petroleum ether extract of leaf. In case of 48 hours the lowest $\mathrm{LD}_{50}$ value was $2.218 \mathrm{mg} / \mathrm{cm}^{2}$ for chloroform extract of root and the highest was $4.535 \mathrm{mg} / \mathrm{cm}^{2}$ also for petroleum ether extract of leaf. And that of 72 hours the lowest $\mathrm{LD}_{50}$ value was 2.024 $\mathrm{mg} / \mathrm{cm}^{2}$ also for the chloroform extract of root and the highest was $3.870 \mathrm{mg} / \mathrm{cm}^{2}$ for petroleum ether extract of leaf (Table 4).

Among the petroleum ether, chloroform, ethyl acetate and methanol extracts of leaf, stem and root the highest percentages of mortality was found in chloroform extract i.e. $73.3 \%$, $70.0 \%$ and $80.0 \%$ respectively in 24 hours and that was for 48 and 72 hours the highest percentages were also for chloroform extracts i.e. $80.0 \%, 76.7 \%, 90.0 \%$ and $90.0 \%, 86.7 \%$, $96.7 \%$ respectively for leaf, stem and root (Table 5).

Mortality of Macrotermes species caused by the root extract of Mimosa diplotricha was high and observed to be concentration and exposure time dependent. The highest concentration $[10 \%(\mathrm{w} / \mathrm{v})]$ of $M$. diplotricha root extract accounted for $100 \%$ mortality against Macrotermes species after a 36 hour exposure period. Following a 36 hour exposure period, the median lethal concentration $\left(\mathrm{LC}_{50}\right)$ of $M$. diplotricha against the termites was $4.12 \%(\mathrm{w} / \mathrm{v})$ (Uyi et al., 2018). In our study we found the related results i.e. root extracts were most effective and $96.7 \%$ mortality was observed by root extract in 72 hour exposure period.

The leaf and stem powders of $M$. diplotricha plant exhibited some degrees of mortality against Sitophilus zeamais Motschulsky which was both concentration and exposure time dependent. At the highest concentration $(3.5 \mathrm{~g})$, the leaf and stem powders of $M$. diplotricha accounted for only $28 \%$ mortality of S.zeamais after a five hour exposure period (Uyi and Samugana 2018). In our study the chloroform extracts showed the highest percent mortality among the four solvent extracts of leaf, stem and root and the root extracts were the most potential in all the three exposure time i.e. 24,48 and 72 hours.

\section{Repellent activity test of $C$. pusillus}

The repellent activity test was performed by following the surface film application method with doses of $314.56,157.28,78.64,39.32$ and $19.66 \mu \mathrm{g} / \mathrm{cm}^{2}$ on half-disc filter paper. The data was recorded with one hour interval for up to five consecutive hours of exposure and the percentage repulsion data was then subjected to ANOVA after transforming into arcsine percentage values. The tables showed that the F-values were 49.74491, 13.11165, 19.99361 and 46.44371 for the analysis between doses and 17.07599, 2.392082, 7.784918 and 16.37612 for the analysis between time interval for leaf extracts of petroleum ether, chloroform, ethyl acetate and methanol (Table 6). In case of stem the Fvalues were 13.4846, 9.968747, 36.56298 and 20.1373 for the analysis between doses and 9.635567, 3.792788, 18.23568 and 13.14133 
for the analysis between time interval for petroleum ether, chloroform, ethyl acetate and methanol extracts (Table 7) and that of the root were $189.6542,66.00051,102.7792$ and 127.6997 for the analysis between doses and 83.48169, 31.1232, 46.72803 and 67.27046 for the analysis between time interval (Table 8). Among the extracts tested the petroleum ether extract of root offered the highest repellent activity at $5 \%(\mathrm{P}<0.05)$ level of significance. According to the intensity of repellent activity the result could be arranged in a descending order: Root (petroleum ether) $>$ Leaf (petroleum ether) > Stem (ethyl acetate).

The following results were found during repellent activity test of $C$. pusilluswith extracts of different parts of $M$. pudica using different solvents.

The leaf and stem powders of $M$. diplotricha exhibited some degrees of repellent activity against Sitophilus zeamais Motschulsky (Coleoptera: Curculionidae), although, the repellent activity was a function of both concentration and exposure time. After a 3 hour exposure period, the $3.5 \mathrm{~g}$ of the leaf $(53 \%)$ and stem $(58 \%)$ powders exhibited the highest repellent activity against $S$. zeamais (Uyi and Samugana, 2018). In our study the root extracts showed strong repellent activity than leaf or stem extracts.

The highest concentration $[10 \%(\mathrm{w} / \mathrm{v})]$ of the root extract of Mimosa diplotricha significantly repelled $100 \%$ of Macrotermes species following a $30 \mathrm{~min}$. exposure period (Uyi et al., 2018). In our investigation we observed that the root extracts possessed the higher potentiality (highest 90\% repellency for petroleum ether extract of root).

In conclusion this work was a basic approach to investigate the insecticidal and repellent activities of the medicinal plant $M$. pudica against $C$. pusillus and the study revealed that the plant extract tested had a strong insecticidal and repellent effect against the pest. Leaf, stem and root each part of the plant had some insecticidal and repellent activities, but root extracts possessed a strong bioactive potential. The findings suggested that the certain bioactive components of $M$. pudica had potential that act as a grain protectant and might be exploited for the control of $C$. pusillus in stored grains in an environmentfriendly way.

\section{Acknowledgements}

The researchers are grateful to University Grants Commission for financial support and the Director, Institute of Biological Sciences, University of Rajshahi for providing the necessary laboratory facilities for the completion of this research.

\section{References}

Addisu, S., D. Mohamed and S. Waktole, 2014. Efficacy of botanical extracts against termites, Macrotermes spp., (Isoptera: Termitidae) under laboratory conditions. Int. J. Agric. Res., 9: 60-73.

Adedire, C.O., O.M. Obembe, R.O. Akinkurolele and S.O. Oduleye, 2011. Response of Callosobruchus maculatus (Coleoptera: Chrysomelidae: Bruchinae) to extracts of cashew kernels. J. Plant Dis. Prot. 118, 75-79.

Adhikarimayum, H., Kshetrimayum, G., Maibam, D. 2010. Evaluation of antioxidant properties of some medicinal plants by sulfur free radical reactivity with curcumin as reference. J Environ, Agric Food Chem.9, 33744.

Banwo, O. and Adamu, R. 2003. Insect pest management in African Agriculture: 
challenges in the current millennium. Arch. Phytopathol. Plant Prot. 36, 5968.

Berenbaum, M.R. 1995. Bugs in the system: Insects and their impact on human affairs. Perseus Publishing; 377.

Bughio, F.M. and Wilkins, R.M. 2004. Influence of malathion resistance status on survival and growth of Tribolium castanium (Coleoptera: Tenebrionidae), when fed on flour from insect-resistant and susceptible grain rice cultivars. J. Stored Prod. Res.40, 65-75.

Chaubey, M.K., 2017. Study of insecticidal properties of garlic, Allium sativum (Alliaceae) and Bel, Aegle marmelos (Rutaceae) essential oils against Sitophilus zeamais L. (Coleoptera: Curculionidae). J. Entomol. 14, 191198.

Cotton, R. T. 1963. Pests of Stored Grain and Grain Products. Burgess Publ. Co., Minneapolis. $318 \mathrm{p}$

Currie, J. E. 1967. Some effects of temperature and humidity on the rates of development, mortality and oviposition of Cryptolestespusillus (Schonherr) (Coleoptera, Cucujidae). J. Stored Prod. Res.3, 97-108.

David, R. (2004). Insect of Stored Products. CSIRO publishing, $192 \mathrm{pp}$.

Fouad, H. Ä., Faroni, L. R. D., Tavares, W. D., Ribeiro, R. C., Freitas, S. D., Zanuncio, J. C. 2014. Botanical extracts of plants from the Brazilian cerrado for the integrated management of Sitotroga cerealella (Lepidoptera: Gelechiidae) in stored grains. J. Stored Prod. Res.57, 6-11.

Howe, R. W., and L. P. Lefkovitch. 1957. The distribution of the storage species of Cryptolestes (Col., Cucuijidae). Bull. Ent. Res. 48,795-809.

Huxtable, R. J. 1992. The pharmacology of extinction. J. Ethnopharm.37, 1-11.
Isman, M. B. 2006. Botanical insecticides, deterrents and repellents in modern agriculture and an increasingly regulated world. Ann. Review Entomol.51, 45-66.

Jha, N. K. Mimosa pudica: Lajjalu. Phytopharm. 2007; 8: 3-8.

Kedia, A., Prakash, B., Mishra, P. K., Singh, P., Dubey, N. K. 2013. Botanicals as eco friendly biorational alternatives of synthetic pesticides against Callosobruchus spp. (Coleoptera: Bruchidae) - a review. J. Food Sci. Technol. 51, 2210-2215.

Khalequzzaman, M., Khanam, L. A. M. and Talukdar, D. 1994: Growth of Tribolium conjugum Duval on wheat flour with various yeast levels. Int. Pest Cont. Magazine36, 128-130.

Khare, C. P. Encyclopedia of Indian Medicinal Plants. Germany: Springer; 2004. pp. 313-4.

McDonald, L. L., Guy, R. H. and Speirs, R. D. 1970. Preliminary evaluation of new candidate materials as toxicants, repellents and attractants against stored-product insects. Agricultural Research Service, US Department of Agriculture, Washington DC. Marketing Research Report no 882.

Melo, B. A., Molina-Rugama, A.J., Leite, D. T., de Godoy, M. S., de Araüjo, E. L. 2014. Bio atividade de pös de espécies végétais sobre a reproduçâo de Callosobruchus maculatus (Fabr. 1775) (Coleoptera: Bruchidae). Bioscience Journal30,346 353.

Mohan, G., Anand, S. P., Doss, A. 2011. Efficacy of aqueous and methanol extracts of Caesalpinia sappan L. and Mimosa pudica $\mathrm{L}$. for their potential antimicrobial activity. South Asian J. Biological Sci. 1, 48-57.

Musah, Rabi A.; Lesiak, Ashton D.; Maron, Max J.; Cody, Robert B.; Edwards, David; Fowble, Kristen; Dane, A. 
John; Long, Michael C. (December 9, 2015). "Mechanosensitivity Below Ground: Touch-Sensitive SmellProducing Roots in the "Shy Plant," Mimosa pudica L". Plant Physiology. 170 1075-1089. doi:10.1104/pp.15.01705.

Nazeema, T. H., Brindha, V.2009. Antihepatotoxic and antioxidant defense potential of Mimosa pudica. Int J Drug Disc. 1, 1-4.

Pascual-Villalobos, M. J. and Robledo, A. 1999. Anti-insect activity of plant extracts from the wild flora in southeastern Spain. Biochem. Systema. and Ecology27, 1-10.

Paul, U. V., Lossini, J. S., Edwards, P. J., Hilbeck, A.. 2009. Effectiveness of products from four locally grown plants for the management of Acanthoscelides obtectus (Say) and Zabrotes subfasciatus (Boheman) (both Coleoptera: Bruchidae) in stored beans under laboratory and farm conditions in northern Tanzania. J. Stored Prod. Res.45, 97-107.

Pavela, R. 2007. Possibilities of botanical insecticide exploitation in grain protection. J. pest Technol. 1, 47-52.

Payne, N. M. 1946. Life history and habits of the flat grain beetle (Laemophloeus minutus Oliv.). J. N. Y. Ent. Soc. 54, 9-12.

Regnault-Roger, C., Vincent, C., Arnason, J. T. 2012. Essential oils in insect control: low-risk products in a highstakes world. Ann. Rev. Entomol.57: 405-424.

Sadia, A. C., Jannatul, I., Rahaman, M. M., Rahman, M. M., Nowshin, N. R., Rebeka, S., 2008. Cytotoxicity, Antimicrobial and Antioxidant Studies of the Different Plant Parts of Mimosa pudica. Stamford J Pharm Sci. 1, 804.

Samuel G, Conor K, Ankit S, M. Mukhlesur
R, Gadria M.M.S, Poonam N2008. Comparative bioactivity studies on two Mimosa species. Boletin Latinoamericano y del Caribe de Plantas Medicinales y Aromaticas 7, 38-43.

Silva, M.L., Silva, L. B., Fernandes, R. M., Lopes, G. S. 2013. Efeito do extrato aquoso e etanölico do angico preto sobre larvas de Rhipicephalus (Boophilus) microplus. Arquivo Brasileiro de Medicina Veterinéria e Zootecnia65, 637-644.

Singh, K., Kumar, A., Langyan, N., Ahuja, M.2009 Evaluation of Mimosa pudica Seed Mucilage as Sustained Release Excipient. AAPS Pharm Sci Tech. 10,1121-7.

Sreelatha, C.and Geetha, P. R. 2011. Pesticidal effects of Clerodendron infortunatum on the fat body of Oryctes rhinoceros (Linn.) male. Journal of Biopesticides. 4, 13-17.

Subramanyam, B. and Hangstrum, D. 1995. Resistance measurement and management. In: Integrated management of insects in stored products (Subramanyam B and Hangstrum D Eds.). Marcel Dekker Inc., New York. pp 331-398.

Talukder, F. A. and Howse, P. E. 1993. Deterrent and insecticidal effects of extracts of pitraj, Aphanamixis polystachya (Meliaceae), against Tribolium castaneum in storage. J. Chem. Ecol. 19, 2463-2471.

Talukder, F. A. and Howse, P. E. 1995. Evaluation of Aphanamixis polystachya as a source of repellents, antifeedants, toxicants and protectants in storage against Tribolium castaneum (Herbst). J. Stored. Prod. Res.31, 55-61.

Tavares, W. D., Grazziotti, G. H., de Souza, A. A., Freitas, S. D., Consolaro, H. N., Ribeiro, P. E. D., Zanuncio, J. C. 
2013. Screening of extracts of leaves and stems of Psychotria spp. (Rubiaceae) against Sitophilus zeamais (Coleoptera: Curculionidae) and Spodoptera frugiperda (Lepidoptera: Noctuidae) for maize protection. J. Food Protect. 76, 1892-1901.

Tavares, W. D., Faroni, L. R. D., Ribeiro, R. C., Fouad, H. A., Freitas, S. D., Zanuncio, J. C. 2014. Effects of astilbin from Dimorphandra mollis (Fabaceae) flowers and Brazilian plant extracts on Sitophilus zeamais (Coleoptera: Curculionidae). Florida Entomologist, 97, 892-901.

Uyi, O. and Samugana, J. 2018. Repellent and Insecticidal Efficacy of Leaf and Stem Powders of Mimosa diplotricha C. Wright ex Sauvalle (Mimosaceae) Against maize weevil Sitophilus zeamais Motschulsky (Coleoptera: Curculionidae). Nigeria Journal of Entomology. 34, 99-107.

Uyi, O., Adetimehin, A. D. and Ogu, O. P. 2018. Repellent and insecticidal activities of the root extracts of Chromolaena odorata and Mimosa diplotricha against Macrotermes species. J. Entomol.15, 135-142.

Uyi, O.O. and B.N. Obi., 2017. Evaluation of the repellent and insecticidal activities of the leaf, stem and root powders of siam weed (Chromolaena odorata) against the Cowpea Beetle, Callosobruchus maculatus. J. Applied Sci. Environ. Manage.21, 511-518.

Uyi, O.O. and G. Osarieme, 2016. Repellence and toxicological activity of the root powder of an invasive alien plant, Chromolaena odorata (L.) (Asteraceae) against Callosobruchus maculatus (Fab.) (Coleoptera: Chrysomelidae). Anim. Res. Int.13, 2510-2517.

Yuan, K., Jia, A., Lu, J. L., Zhu, J. X. 2007. Structural identification of new Cglycosylflavones from Mimosa pudica. Chin J An Chem. 35, 739-742.

Yuan, K., Jia, A., Lu, J. L.2006. Isolation and Identification of phenolic constituents from Mimosa pudica. Chin J Chin Materia Medica 31, 1029-30.

Yuan, K., Lu, J. L., Jia, A., Zhu, J. X.2007. Two new C-glycosyl flavones from Mimosa pudica. Chin Chem Lett.18, $1231-4$.

Yuan, K., Lu, J. L., Jia, A.2006. Studies on Chemical Constituents of Mimosa pudica. Chin Pharm J.41,1293-5.

Yuan, K., Lu, J. L., Yin, M. W.2006. Chemical constituents of C-glycosyl flavones from Mimosa pudica.Yao XueXuebao. 41, 435-8.

\section{How to cite this article:}

Ujjwal Kumar Mondol and Islam, W. 2020. Insecticidal and Repellent Activities of Mimosa pudica L. (Fabaceae) against Cryptolestes pusillus (Schon) (Coleoptera: Cucujidae). Int.J.Curr.Microbiol.App.Sci. 9(09): 2222-2235. doi: https://doi.org/10.20546/ijcmas.2020.909.277 